\title{
Towards an Aero-Propulso-Servo-Elasticity Analysis of a Commercial Supersonic Transport
}

\author{
Joseph W. Connolly, George Kopasakis ${ }^{\dagger}$ \\ NASA Glenn Research Center, Cleveland, OH 44135, USA

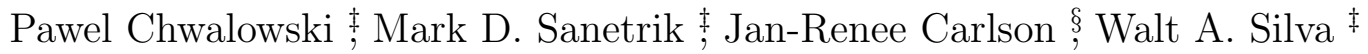 \\ NASA Langley Research Center, Hampton, VA 23681, USA \\ and \\ Jack McNamara \\ The Ohio State University, Columbus, $\mathrm{OH}$ 43210, USA
}

\begin{abstract}
This paper covers the development of an aero-propulso-servo-elastic (APSE) model using computational fluid dynamics (CFD) and linear structural deformations. The APSE model provides the integration of the following two previously developed nonlinear dynamic simulations: a variable cycle turbofan engine and an elastic supersonic commercial transport vehicle. The primary focus of this study is to provide a means to include relevant dynamics of a turbomachinery propulsion system into the aeroelastic studies conducted during a vehicle design, which have historically neglected propulsion effects. A high fidelity CFD tool is used here for the integration platform. The elastic vehicle neglecting the propulsion system serves as a comparison of traditional approaches to the APSE results. An overview of the methodology is presented for integrating the propulsion system and elastic vehicle. Static aeroelastic analysis comparisons between the traditional and developed APSE models for a wing tip deflection indicate that the propulsion system impact on the vehicle elastic response could increase the deflection by approximately ten percent.
\end{abstract}

\section{Nomenclature}

\begin{tabular}{|c|c|c|c|}
\hline$A$ & Cross-sectional area & $x$ & Length \\
\hline$P$ & Pressure & $\gamma$ & Ratio of specific heat \\
\hline$R$ & Gas Constant & $\rho$ & Density \\
\hline$T$ & Temperature & \multicolumn{2}{|c|}{ Subscripts } \\
\hline$V$ & Volume & $s$ & Static flow condition \\
\hline$c_{p}$ & Coefficient of pressure & $t$ & Total flow condition \\
\hline$\dot{m}$ & Mass flow rate & & \\
\hline$t$ & Time & & \\
\hline
\end{tabular}




\section{Introduction}

TASA aims to overcome the obstacles associated with supersonic commercial flight by developing technologies that will allow for a practical supersonic overland commercial transport. The primary driver of this technology development is to reduce noise associated with the sonic boom. Thus, the proposed vehicles are long, slim body aircraft with the potential for pronounced structural vibrations that will need to be controlled. This phenomena of aerodynamic forces inducing structural vibrations is known as aeroelasticity. The controlling of these structural vibrations is known as aero-servo-elasticity (ASE). NASA has investigated the ASE problem extensively using both computational and experimental methods in both the subsonic and supersonic flight regimes. ${ }^{1}$

The primary advancement to the ASE field presented here is the integration of a nonlinear dynamic model of a propulsion system into an elastic vehicle model. The propulsion system is comprised of an axisymmetric external compression inlet, variable cycle turbofan engine (VCE), and convergent-divergent (CD) nozzle. The vehicle is a commercial supersonic transport larger in scale than the Concorde, a previous generation commercial supersonic transport. The combination of the ASE and propulsion system will be referred to as aero-propulso-servo-elasticity (APSE). APSE considerations can impact aircraft performance such as ride quality and stability, leading to vehicle design challenges. Furthermore, if the inclusion of the propulsion system has a significant impact on the structural modes of the vehicle, then the sonic boom signature could be impacted. As the boom signature is the primary obstacle to commercial supersonic flight, this new computational tool is expected to play an important role in future vehicle designs.

Previously, the major components of the propulsion system used for this study were developed ${ }^{2}$ and aeroelastic studies of the supersonic transport ${ }^{3}$ were conducted. This paper leverages this past work to investigate the vehicle elastic deformations of the coupled propulsion system and vehicle using higher fidelity computational fluid dynamic (CFD) modeling. This higher fidelity modeling will capture thrust, structural dynamics, and aerodynamics as required for the APSE task. Typically, the propulsion impacts for elastic vehicle studies in the subsonic and supersonic flight regimes have been ignored. Recently there has been efforts to understand the coupling between the propulsion system and subsonic flight vehicles. ${ }^{4-7}$ The previous work has focused on simple physics models to understand the basic coupling, with some considerations for notional thrust perturbations. ${ }^{8}$ Greater consideration for the coupling of the propulsion system and vehicle have been considered for hypersonics vehicles where the scramjet propulsion system and vehicle are tightly integrated. ${ }^{9-11}$

This paper is organized as follows: first, an overview will be provided of the APSE model along with a description of the structural and propulsion system component models of a commercial supersonic transport vehicle. Next, the computational modeling approach to interface these models will be discussed for the overall APSE simulation. The changes from the typical ASE computational approach to enable the APSE model are highlighted. Finally, the paper will outline APSE modeling results, future work, and conclusions.

\section{APSE Model Overview}

The overall APSE model block diagram can be seen in Fig. 1. The propulsion system, highlighted in Fig. 1, is comprised of the external compression inlet, VCE with fuel flow controller, and CD nozzle. The linear aeroelastic vehicle impacts are based on a finite element model (FEM) analysis of the vehicle. Both the vehicle and propulsion system models are directly impacted by the freestream flight conditions, which have the capability to be time varying to simulate atmospheric turbulence or alteration of the vehicle angle of attack. The propulsion system and aeroelastic vehicle have two primary coupling interactions. The first being variations of the vehicle or propulsion system that impact the surrounding flow field. This includes vehicle wing vibrations that subsequently induce perturbations into the flow field in front of the mounted propulsion system, the propulsion system inlet shock field, and nozzle exit plume impact on the vehicle. The flow field perturbations are simulated within the CFD calculations and are illustrated in Fig. 1 with bold arrows to identify information flow direction. The second APSE coupling interaction is the thrust perturbations that have the potential to accentuate existing structural modes through the forces exerted on the vehicle structure. 


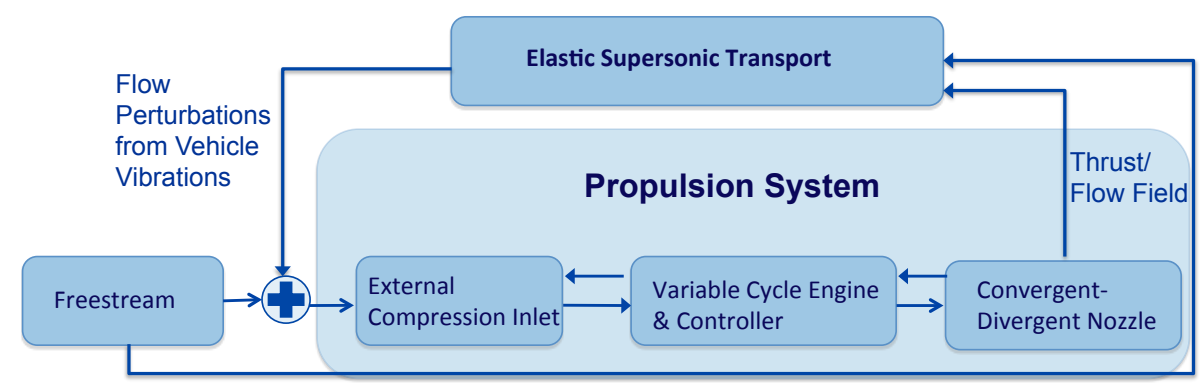

Figure 1. Block diagram of overall APSE system model with propulsion system highlighted.

\section{A. N+2 Commercial Supersonic Transport Model}

To address future challenges in aerospace, NASA has a technology development approach to advance modeling tools and capabilities to meet the requirements for future generations of aerospace vehicles. Under the NASA two generations from present state $(\mathrm{N}+2)$ design, Lockheed Martin has developed the low-boom supersonic configuration illustrated conceptually in Fig. $2 .^{3}$ The key features to note for this study are the long slender vehicle profile and the three gas turbine engines. Two of the engines are mounted under the wing, with a third mounted in the back along the centerline of the fuselage. The two engines mounted under the wing are close to the fuselage, which may offer some relief from possible aeroelastic problems as opposed to having the engines being mounted further out on the wing. However, having these large masses located near the tail section of a flexible fuselage may lead to aeroelastic problems that could impact ride quality. ${ }^{3}$
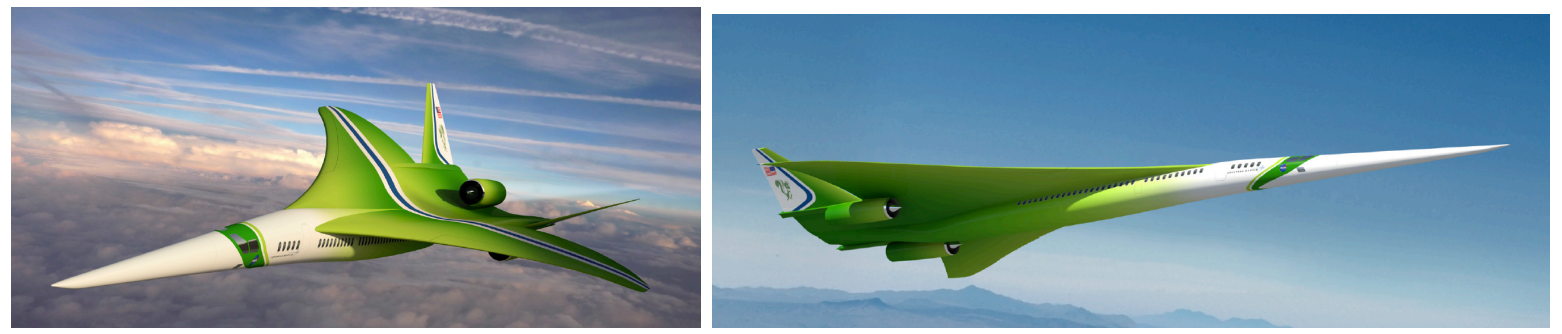

Figure 2. Artistic concept of the Lockheed Martin N+2 commercial supersonic transport vehicle.

The general characteristics of the $\mathrm{N}+2$ configuration are listed in Table 1 . As a comparison of the $\mathrm{N}+2$ configuration to the previous supersonic commercial transport, the Concorde, the $\mathrm{N}+2$ configuration is about $21 \%$ longer with approximately the same wingspan. The primary difference is the lower cruise Mach number of 1.7 compared to that of the Concorde cruise Mach number of two. One of the drivers for the lower flight speed is to reduce the complexity of lowering the sonic boom noise signature, to allow supersonic flight over land.

Table 1. The basic size, weight, and cruise operating condition of the Lockheed Martin concept N+2 commercial supersonic transport.

\begin{tabular}{|c|c|c|}
\hline \multicolumn{3}{|c|}{ Geometry } \\
\hline \hline $\begin{array}{c}\text { Length } \\
244 \mathrm{ft}\end{array}$ & $\begin{array}{c}\text { Span } \\
83 \mathrm{ft} \mathrm{10} \mathrm{in}\end{array}$ & $\begin{array}{c}\text { Height } \\
30 \mathrm{ft} 6 \mathrm{in}\end{array}$ \\
\hline \multicolumn{3}{|c|}{ Weight } \\
\hline \hline Take Off & Fuel & Empty \\
\hline $320,000 \mathrm{lbs}$ & $168,000 \mathrm{lbs}$ & $136,000 \mathrm{lbs}$ \\
\hline \multicolumn{3}{|c|}{ Cruise Operating Condition } \\
\hline \hline Altitude & Mach & Angle of Attack \\
\hline $50,000 \mathrm{ft}$ & 1.7 & 2.25 deg. \\
\hline
\end{tabular}




\section{Vehicle Dynamic Model}

The vehicle dynamic model was developed using an FEM that incorporates all representative structural components. The FEM was developed for a composite and metallic layout with structural design constraints based on landing, maneuvering, and gust loads, as well as strain, buckling, and minimum gauge criteria. The FEM was used to carry out a normal modal analysis to identify the relevant modes for the CFD-based aeroelastic analysis.

The majority of the FEM was discretized into constant property design zones, and an optimizer was used to adjust the structural properties using the design variables defined within these zones. ${ }^{3}$ Skins assumed a sandwich approach consisting of graphite and Bismaleimide (BMI) unidirectional tape with a honeycomb core, resulting in three independent design variables per zone ( either 0,45 , or 90 degree plies; core thickness remains constant in sizing). The design of the substructure also assumed a sandwich approach with graphite and BMI fabric facesheets. Modeling the substructure assumed a quasi-isotropic laminate, resulting in one design variable per zone. Core thickness for the substructure also remained constant during sizing. A representative mass distribution was also developed and applied that accounted for systems, payload, and various fuel states. Buckling was addressed during optimization by coupling an in-house analysis code along with the MacNeal-Schwendler Corporation (MSC) NASA structure analysis (NASTRAN) code. ${ }^{12}$ The mixed metallic and composite material configuration is representative of modern aircraft. The $\mathrm{N}+2$ concept skin composition percentages for this study: composites $55 \%$, aluminum $26 \%$, titanium $16 \%$, and steel $3 \%$. The FEM has roughly 170,000 degrees of freedom.

A modal solution was performed on the FEM taking advantage of symmetry while defining the boundary conditions. The first four flexible symmetric modes are shown in Fig. 3. Not shown are the three rigid body modes. More details can be found in previous reports. ${ }^{3}$ The first 22 flexible modes from this analysis are used for the CFD based static aeroelastic analysis.

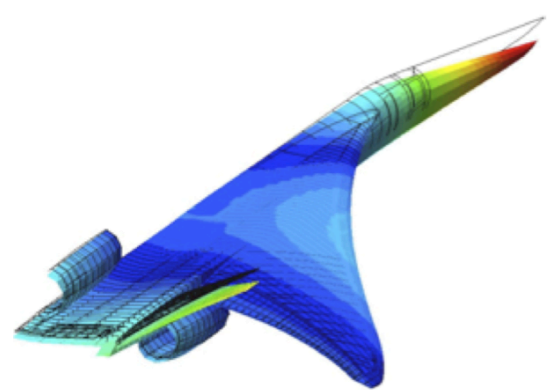

(a) Mode $4(2.23 \mathrm{~Hz})$ : Fuselage bending

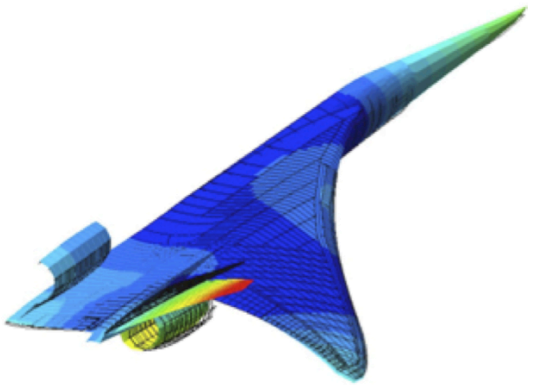

(c) Mode $6(3.38 \mathrm{~Hz})$ : Tail bending

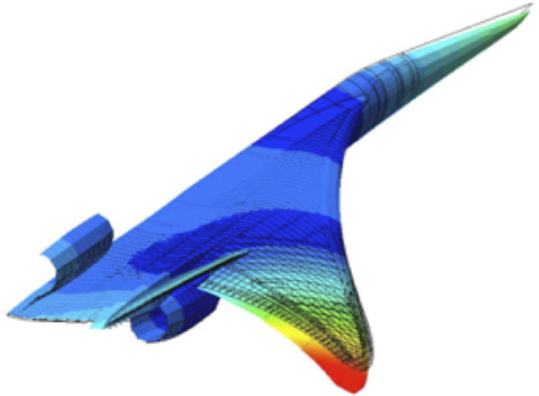

(b) Mode $5(2.44 \mathrm{~Hz})$ : Wing bending

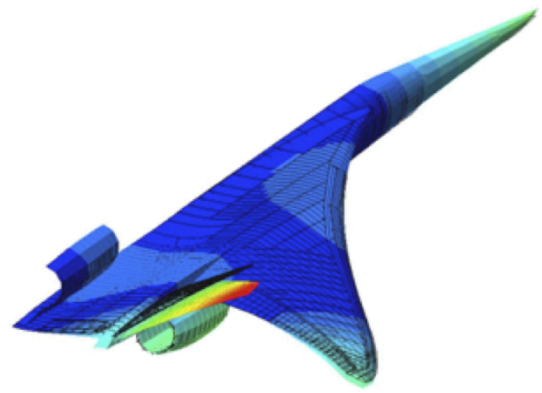

(d) Mode $7(3.67 \mathrm{~Hz})$ : Wing-Tip bendingtorsion, fuselage 2 nd bending

Figure 3. First four flexible modes of the vehicle with hollow engine nacelles finite element analysis.

\section{B. Propulsion System Model}

The propulsion system for this study includes an external compression inlet, VCE, and CD variable geometry nozzle. Previously, the major components of the propulsion system used for this study were developed 
separately. ${ }^{13}$ This paper leverages this past work to investigate the integration of the lumped volume approach for the VCE into an aeroelastic vehicle model. This level of modeling fidelity for the key engine components of the propulsion system will allow for accurate representation of the thrust dynamics. Integrated propulsion system one-dimensional dynamic models have been developed in support of previous NASA supersonic projects by Garrard, ${ }^{14-16}$ Gamble, ${ }^{17}$ Numbers, ${ }^{18}$ and Giannola. ${ }^{19}$ The distinction in the work presented here is that the main goal is to provide a platform for integration into a high fidelity ASE vehicle model. As such, the external components of the propulsion system are modeled using a high fidelity CFD tool, whereas the turbomachinery will be modeled using the previously developed lumped volume approach.

The engine is the primary component of the overall propulsion system, and its turbomachinery model is of primary interest to this study. This turbomachinery model is integrated into the CFD tool. The current engine design concept of the supersonic propulsion system is shown in Fig. 4. Captured airflow coming from the inlet passes through the fan component and then splits into three gas paths as illustrated in Fig. 4. The primary gas path, similar to a turbojet, comprises the core of the engine. The secondary gas path, similar to a typical turbofan engine, enables a large amount of the captured airflow to bypass around the core of the engine. The more advanced conceptual part of this engine is the third gas path,

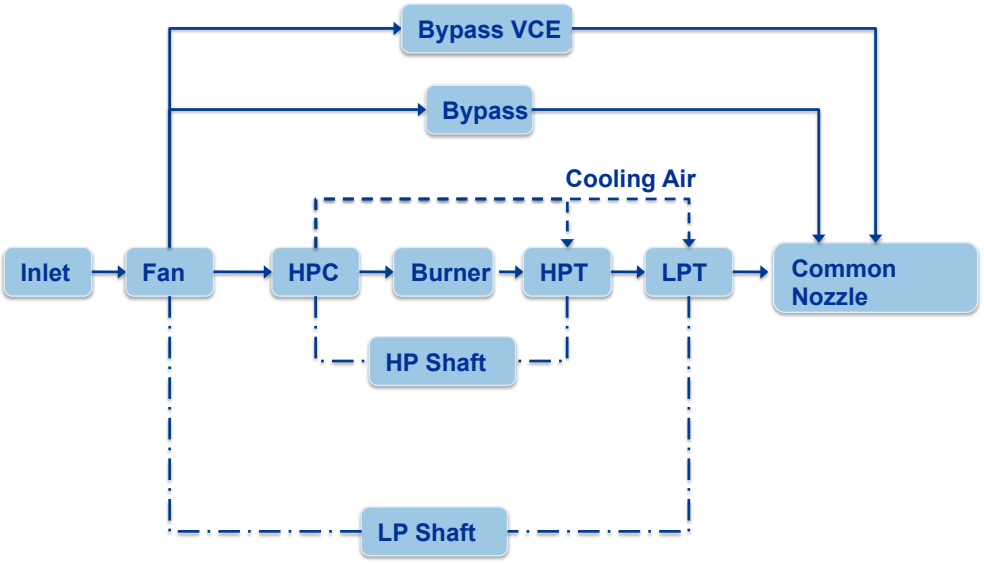

Figure 4. Variable cycle engine primary components modeled as lumped volumes. called the Bypass VCE. This path is designed to provide a lower exit airflow velocity that could be used as a noise shield for the flow exiting the core. In addition, the third flow path provides a means to optimize the thermodynamic cycle throughout the flight envelop. For this study, the three streams will be mixed in a common duct before exiting a single nozzle. This was due to complications of including the propulsion system mesh in the $\mathrm{N}+2$ existing hollow engine mesh. All of the major engine components shown in Fig. 4 are modeled with inviscid conservation equations. The modeling approach was previously developed and verified against available dynamic experimental data and other computational tools. ${ }^{2,20,21}$

\section{Computational Approach}

For the development of the APSE model, NASA Langely's Fully Unstructured Navier-Stokes in Three Dimensions (Fun3D) code $^{22}$ is being used as the integration platform. The Fun3D code has various configuration options to include tools outside of the standard flow solver and is a useful tool for CFD based ASE analysis. ${ }^{3,23}$ All of the analysis presented here is based on solutions of the Euler equations, assuming the airflow is calorically perfect and a compressible gas. Fun3D uses a node-based finite-volume unstructured flow solver. ${ }^{24}$ The method chosen for this study, was a blended scheme that is a selectivelydissipative version of the low-dissipation flux splitting scheme (LDFSS). ${ }^{25}$ The inviscid flux scheme acts as the van Leer scheme at shocks via a shock detector and LDFSS near walls. The inviscid flux limiter used is a stencil based van Albada limiter with a heuristic pressure limiter. ${ }^{26}$ The inviscid flux scheme was chosen for its ability to provide a more stable solution given the normal shocks associated with the inclusion of the propulsion system. The flux limiter method was chosen for its ability to be turned off after a period of simulation iterations that improved the stability of the solution. Prior to running aeroelastic solutions, a steady-state solution was obtained using an Euler implicit backwards difference scheme, with local time stepping to accelerate convergence. The unsteady simulations are calculated using a modified, optimum 2nd-order backward difference formula (BDF) scheme. ${ }^{27}$ 


\section{A. Aeroelastic Analysis}

FUn3D has a dynamic aeroelastic capability for linear structural dynamics using an internal modal solver. ${ }^{28}$ The modal solver is implemented in FUn3D in the same general manner as other NASA CFD codes. ${ }^{29}$ For the computations presented here, the structural modes were obtained via a normal modes analysis with the MSC NASTRAN code that uses FEM numerical analysis. Structural modes were interpolated to the CFD surface mesh using a method developed by Samareh. ${ }^{30}$

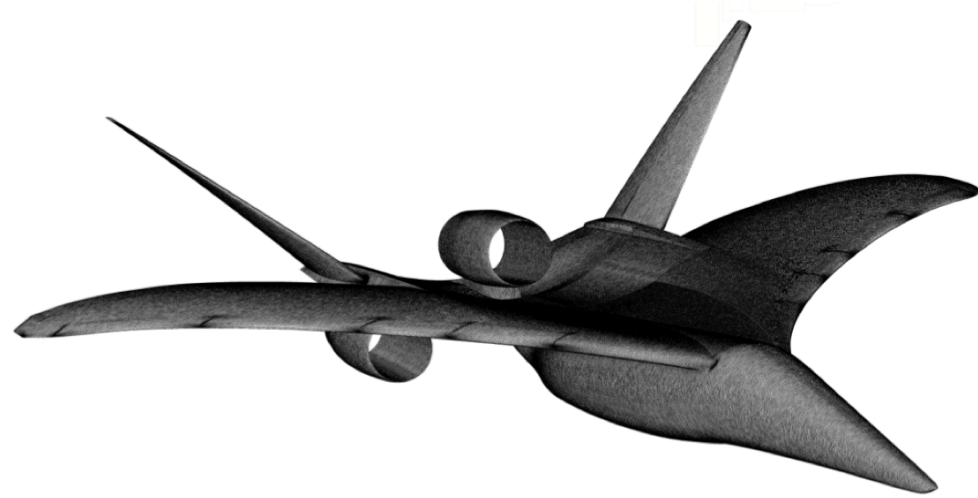

Figure 5. Unstructured grid of the $\mathrm{N}+2$ hollow engine configuration.

Shown in Fig. 5 is an aspect view of the inviscid unstructured surface grid generated for the vehicle with hollow engine nacelles. The symmetric half-plane unstructured surface grid, which is used for the CFD studies, has 298,085 points and 555,710 cells. The grid without the propulsion system is used for static aeroelastic analyses. This static aeroelastic analysis will serve as the traditional approach to compare with the static aeroelastic results for the full APSE model. Initial cruise condition inviscid steady-state Fun3D solutions have been conducted using both a coarse and a fine grid (ten times more points). ${ }^{3}$ Comparison of elastic deformation performance parameters between the two grids indicated only minor differences thereby permitting use of the coarse faster solving grid for most analyses.

\section{B. Aeroelastic Dynamic Model Modifications for APSE}

Several modifications to the above discussed aeroelastic analysis is needed to develop the APSE model. The primary change is the inclusion of the external propulsion system components to the CFD mesh. An axisymmetric external compression inlet and single CD nozzle are added to the previous hollow engine nacelles. This change resulted in the grid shown in Fig. 6. The previous mesh is illustrated as a shaded feature and the additional propulsion components within the engine nacelles are illustrated as a mesh. A closeup with only the propulsion components is also included to visualize the finer details. Adding the propulsion system increased the grid to 3,516,774 points and 22,655,094 cells.

The FEM does not include any of the propulsion system components other than the engine nacelle. Thus, some basic assumptions were made to link the structural modes to the new propulsion element features. Primarily, the engine elements can be considered rigid in relation to the rest of the vehicle. The same interpolation scheme developed by Samareh is used here, with a closest point assumption to interpolate the structural modes to the vehicle mesh with the propulsion system. Boundary planes are added for the inletengine and engine-inlet fluid interfaces. The modes were numerically interpolated to the inlet-engine and engine-nozzle boundary planes to allow for the fluid boundary plane to move with the grid deformations. This reduced runtime problems associated with negative volumes. To illustrate the interpolation of the modes to the new features of the CFD mesh, the fourth mode ' $z$ ' direction displacement is shown in Fig. 7. Only the fourth mode is used here to illustrate the interpolation technique, but all 22 flexible modes are interpolated to the new mesh. 


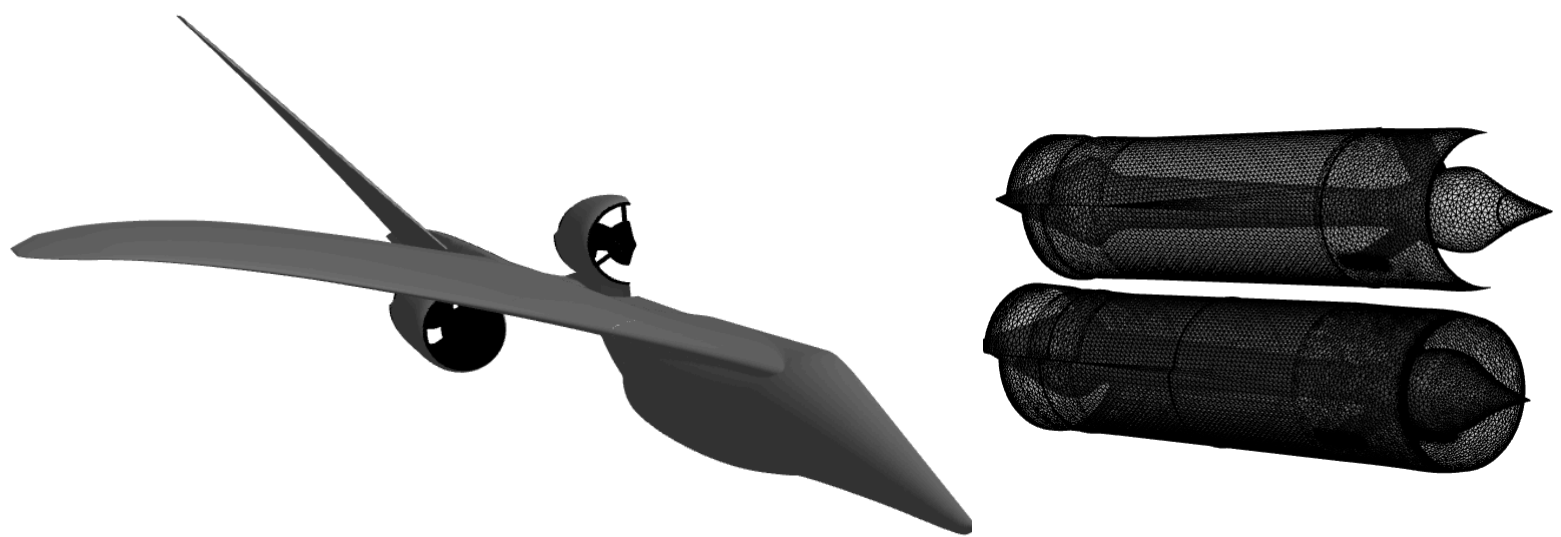

Figure 6. Unstructured grid of the $\mathrm{N}+2$ vehicle with external propulsion system components.

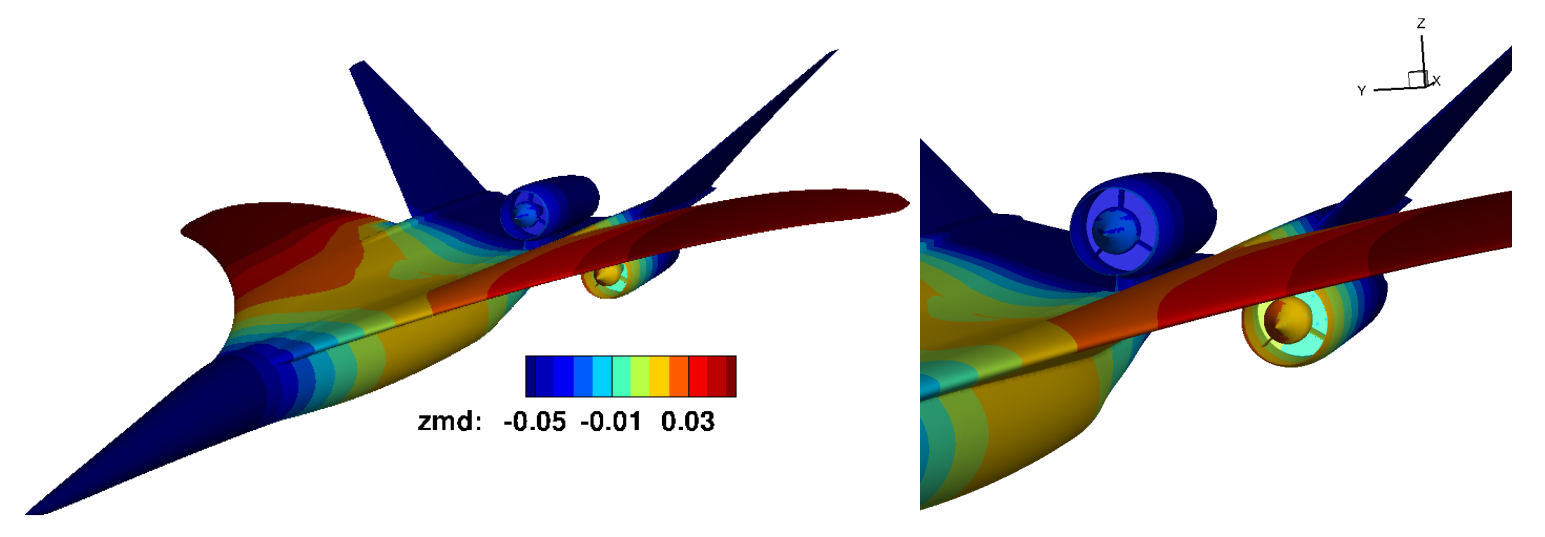

Figure 7. Mode 4: Fuselage bending mode at $2.23 \mathrm{~Hz}$, numerically interpolated to the external propulsion system geometry and with a close up view of the propulsion system.

\section{Aeroelastic - Propulsion Integration}

A key contribution of this work is the integration of the propulsion model into the Fun3D code. Here, the turbomachinery model is included as a stand-alone simulation module, whereas Fun3D models the external inlet and nozzle flows. This will allow for calculating thrust dynamics. ${ }^{2}$

\section{Inlet to Engine Boundary}

The engine simulation requires total pressure and total temperature calculations from the Fun3D subsonic outflow boundary. The coupling approach used standard engine model input boundary conditions with minor changes to the existing subsonic outflow boundary condition in Fun3D. ${ }^{31}$ This approach reduced some of the modifications required in FUn3D. The aerodynamic interface plane (AIP) or the exit boundary condition of the inlet is typically the most stable in Fun3D, if a prescribed static pressure for the subsonic outflow is used. Therefore, in FUN3D the static pressure is specified for this outflow boundary condition and the velocity and temperature are extrapolated from the internal grid points. Since this is a subsonic outflow boundary interface, two flow values are calculated and one is defined at the AIP. To interface with the one dimensional engine code, the three dimensional values are averaged to a single axial value. ${ }^{32}$ These mean values are then used to calculate the total pressure and temperature at the AIP boundary. The engine simulation then uses a calculated mass flow rate from FUn3D at the AIP boundary to update the specified inlet back pressure.

A duct volume is modeled prior to the turbomachinery components in the engine simulation to calculate the described flow variables at the inlet-engine boundary, where the conservation equations for this duct 
volume can be expressed as Eqs. (1) to (3).

$$
\begin{array}{r}
\frac{d}{d t}\left(\rho_{s}\right)=\frac{1}{V}\left(\dot{m}^{\text {inlet }_{\text {exit }}}-\dot{m}^{\text {engine }}\right) \\
\frac{d}{d t}(\dot{m})=\frac{A}{x}\left(P_{s}^{\text {inlet }_{\text {exit }-1}}-P_{s}^{\text {inlet }_{\text {exit }}}\right) \\
\frac{d}{d t}\left(\rho_{s} T_{t}\right)=\frac{\gamma}{V}\left(T_{t}^{\text {inlet }_{\text {exit }-1}} \dot{m}^{\text {inlet }_{\text {exit }}}-T_{t}^{\text {inlet }_{\text {exit }}} \dot{m}^{\text {engine }}\right)
\end{array}
$$

The superscripts used in the above equations indicate the location of the variable relative to the AIP and engine face. The subscript "s" signifies static values and subscript "t" signifies total values. These equations are used in conjunction with the state equation, $P=\rho R T$, to obtain the inlet exit state variables for the downstream engine face and the upstream inlet grid points.

\section{Engine to Nozzle Boundary}

A similar approach to that described for the AIP boundary is used for the engine-nozzle boundary. Here the engine simulation calculates the total pressure and temperature at the engine-nozzle boundary, while calculations pertaining to the mass flow are calculated with the Fun3D nozzle model. The modeling of this interface is accomplished by modifying the Fun3D subsonic inflow boundary condition. For the subsonic inflow boundary condition, two flow values are defined and one is extrapolated from the internal grid points. The three dimensional values from Fun3D are averaged to a single one dimensional axial value for the mass flow. Here the engine simulation defines the total pressure and temperature for the nozzle plenum assuming uniformity across the boundary, while the mass flow information is passed to the engine model from Fun3D. Since the engine simulation is a one dimensional model, any flow distortion in the inlet is averaged out at the nozzle interface. The mass flow coming from Fun3D replaces a previously employed simple choked flow boundary condition in the engine simulation with a very small duct volume added at the exit of the engine simulation. This duct volume is modeled using volume dynamics, in a similar manner to that at the AIP as described, since both interfaces are subsonic.

\section{Results}

This section reveals some initial findings for the $\mathrm{N}+2$ vehicle with APSE impacts included. A cruise flight condition is used for this study of Mach 1.7 at an altitude of 50,000 ft, and an angle of attack of 2.25 degrees. The results will be broken out into two sub-sections: one for the rigid body analysis of the model and the other for the static aeroelastic analysis of the traditional hollow engine modeling approach compared to the APSE model.

\section{A. Rigid Body Analysis}

The full propulsion system has been integrated into the $\mathrm{N}+2$ vehicle and a stable steady-state solution was obtained. However, the steady-state solution did not converge to the same nine orders of magnitude as was obtained with just the hollow engines. The steady-state solution with the propulsion system converged to approximately four orders of magnitude, as shown in Fig. 8. This was because the normal shock of the inlet was oscillating about the

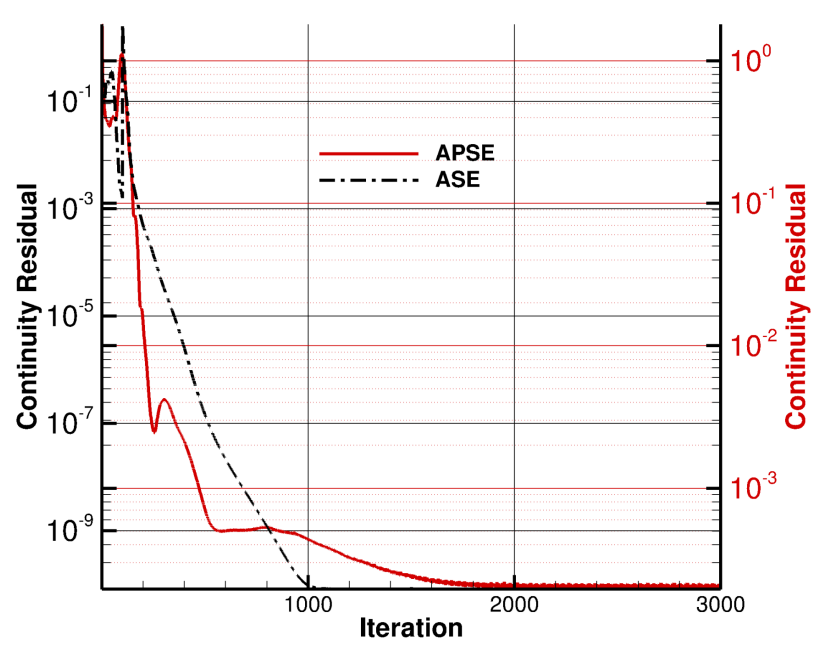

Figure 8. Continuity equation residual convergence for vehicle coupled with propulsion system. internal and external position of the inlet duct. The solution stability can be improved by increasing the inlet back pressure and pushing the terminal shock outside of the inlet; however, this increases the shock 
strength and the impact of the shock on the vehicle. Increased dissipation, as would be expected for a viscous solution helps to alleviate this problem.

To illustrate the terminal shock location that is oscillating and causing some convergence problems for the inviscid solution, a slice of the three dimensional Mach contours is shown in Fig. 9 for the wing mounted engine. The external portions of the inlet and nozzle are included in Fun3D, and the engine simulation connects the inlet to the nozzle as previously discussed. The approximate normal shock locations in Fig. 9 are shown as black bands. The normal shocks are located at the cowl of the inlet and at the aerodynamic throat of the nozzle. While causing some convergence problem for the inviscid solution, these are the expected shock locations for the cruise configuration. As the normal shocks in the inlet move forward, they become stronger and induces greater total pressure losses. In addition, this causes greater forces on the vehicle that can cause elastic deformations.

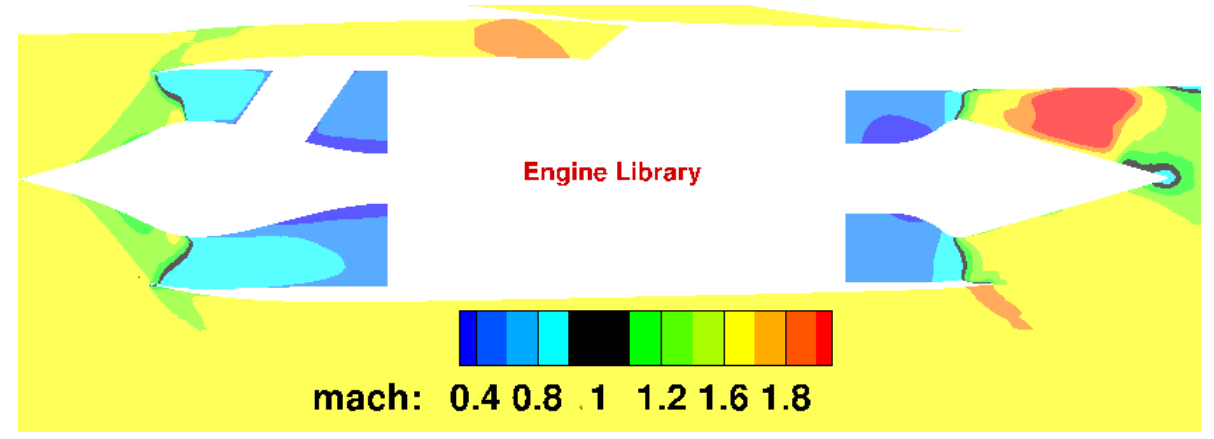

Figure 9. $\mathrm{N}+2$ wing mounted propulsion system with mach contours.

The coefficient of pressure contours on the vehicle for the baseline rigid body are shown on the left side of Fig. 10. The normalized pressure contour for the engine boundary is plotted. A close-up front view of the $\mathrm{N}+2$ vehicle pressure profile is shown on the right side of Fig. 10. One of the interesting features shown in Fig. 10 is the significantly larger amount of distortion entering the engine under the wing. This could be from shocks emanating from the wing, or potential problems at the boundary that could cause the inlet to experience "buzz" or oscillations.

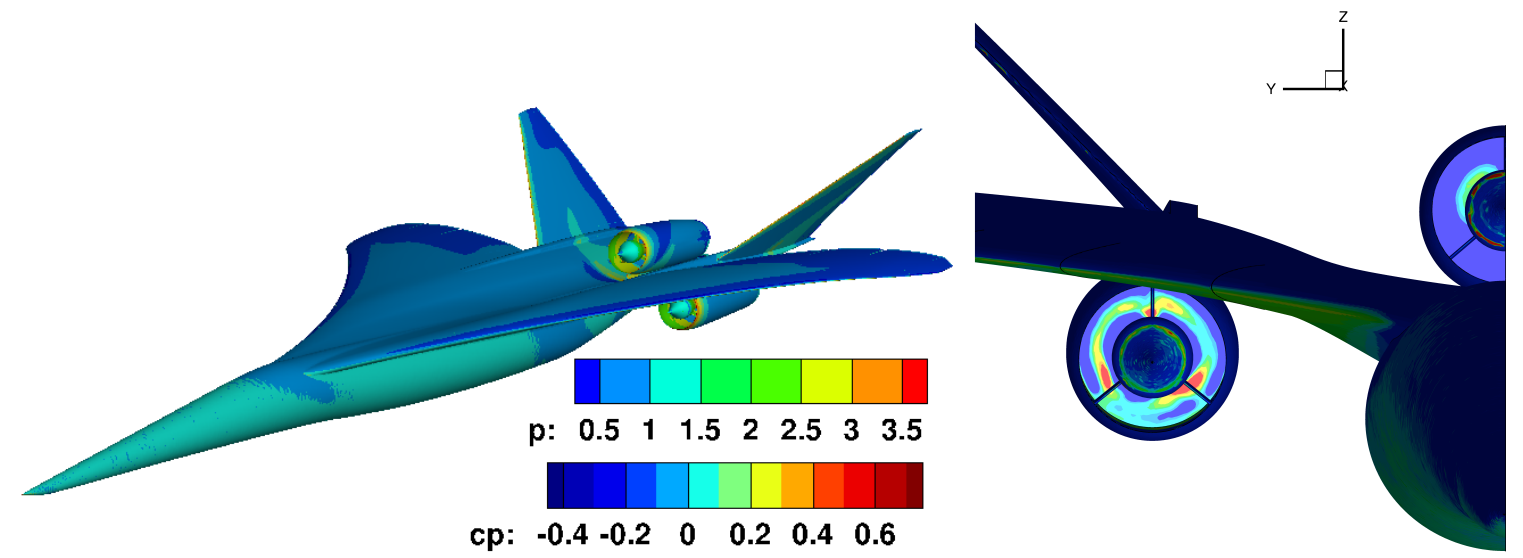

Figure 10. Fully integrated APSE model steady-state coefficient of pressure on vehicle and pressure profile for engines.

\section{B. Static Aeroelastic Analysis}

This section will cover the static aeroelastic response of the $\mathrm{N}+2$ vehicle while including modeling impacts of the propulsion system. However, stability problems arose while restarting from a steady-state solution to run the time accurate static aeroelastic solution with the dynamic engine simulation. While the dynamic engine simulation was able to run to a steady-state solution and in a time accurate mode as illustrated in the 
above section, the current restart feature for the engine simulation still exhibits a transient offset at startup. This transient offset in conjunction with the start of the aeroelastic deformations is causing the simulation to become unstable. While work is ongoing to resolve the restart problem, the engine simulation function was used to determine the inlet back pressure, nozzle total pressure, and total temperature to define the engine boundaries. For the results presented in this section, the boundary values are held fixed to stabilize the CFD solution.

Holding the engine boundary values to a fixed value will effectively provide an approximate steady-state thrust for the propulsion system. Thus, the results in the following section will effectively provide a best case scenario, where perturbations will not be able to significantly impact the position of the normal shock. This limits the normal shock increase in strength as it moves off of its design point, limiting the propulsion systems impact to deform the vehicle body.

Traditionally, when doing an aeroelastic study, the propulsion system is not included. The results here compare the traditional approach with the hollow engine nacelles to that of the vehicle with the propulsion system using fixed boundary conditions. The delta coefficient of pressure is the difference in the coefficient of pressure from the rigid body baseline to that of the elastically deformed body. Contours of the delta coefficient of pressure on the vehicle are shown in Fig. 11 as a top view, and are used as a first indication of potential differences between an ASE and APSE model. A plane of symmetry from the nose to the tail is used in Fig. 11 such that the left side of vehicle shows aeroelastic results with a hollow engine, and the right shows the results including the propulsion system. While the results look very close to being a mirror image, the contours are not symmetric illustrating variations in the flow field surrounding the vehicle.

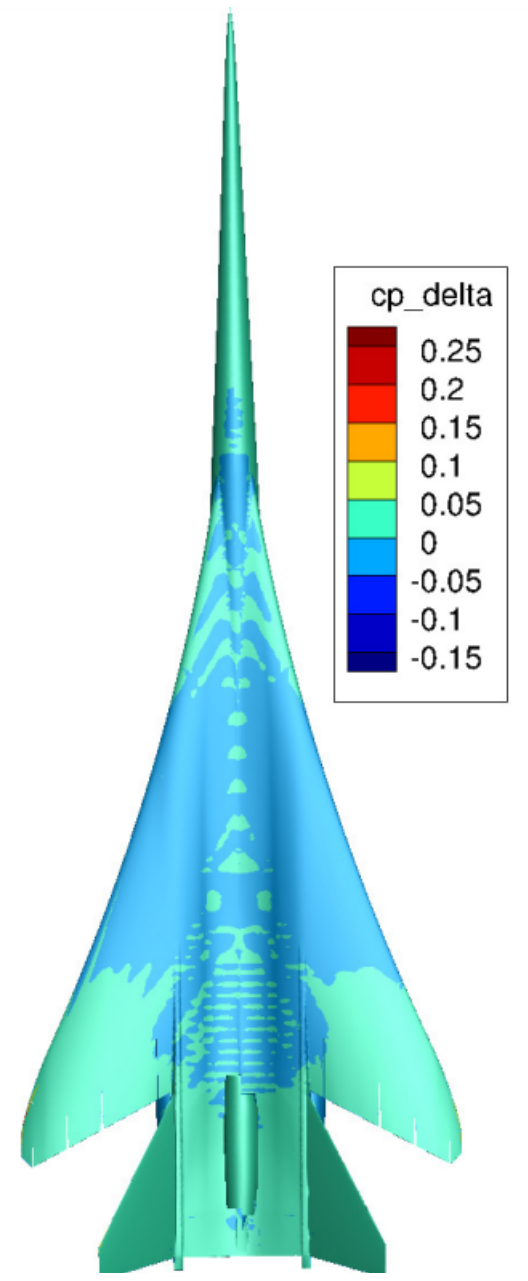

Figure 11. A top view of the difference in the coefficient of pressure contours from the rigid body to the elastically deformed body. The full vehicle image shows both the ASE and APSE results. The left portion of the vehicle shows aeroelastic impacts and the right shows the aero-propulso-elastic.

The static aeroelastic solution was obtained using the previously discussed 22 flexible modes numerically 
interpolated to the hollow engine grid and also to the grid including the propulsion elements. The CFD mesh of the baseline rigid body (black) is shown in Fig. 12, along with the aeroelastic (gray) and aero-propulsoelastic (red) vehicle deformations. The full vehicle is shown in the top left of Fig. 12, whereas a close up wing tip deflection is shown in the right and bottom. The actual deflections at the wing tips are relatively small. The traditional approach with the hollow engine nacelles has a wing tip deflection of approximately 6.6" compared to the rigid body. The inclusion of the propulsion system impacts, albeit somewhat limited in the current study, showed a deflection of 7.3". This amounts to about a $10 \%$ under prediction of the elastic response of the vehicle when neglecting the impacts of the propulsion system. As was stated before, this is under ideal conditions and the results could increase in the fully integrated APSE model. These results may help define error bounds for the vehicle structural deformation to support sonic boom calculations, which are critical to ensuring that future commercial supersonic vehicles can meet potential noise regulations.

While the final APSE model could illustrate larger deflections due to shock movement, a final flight vehicle would likely alter designs to ensure normal shock stability about the design point. However, with an accurate APSE model, adjustments to obtain a stable cruise design point could be obtained earlier in the design phase instead of later during flight tests. Adjustments after flight tests can be costly, or could increase complexity if an active inlet control system is required to stabilize the normal shock.

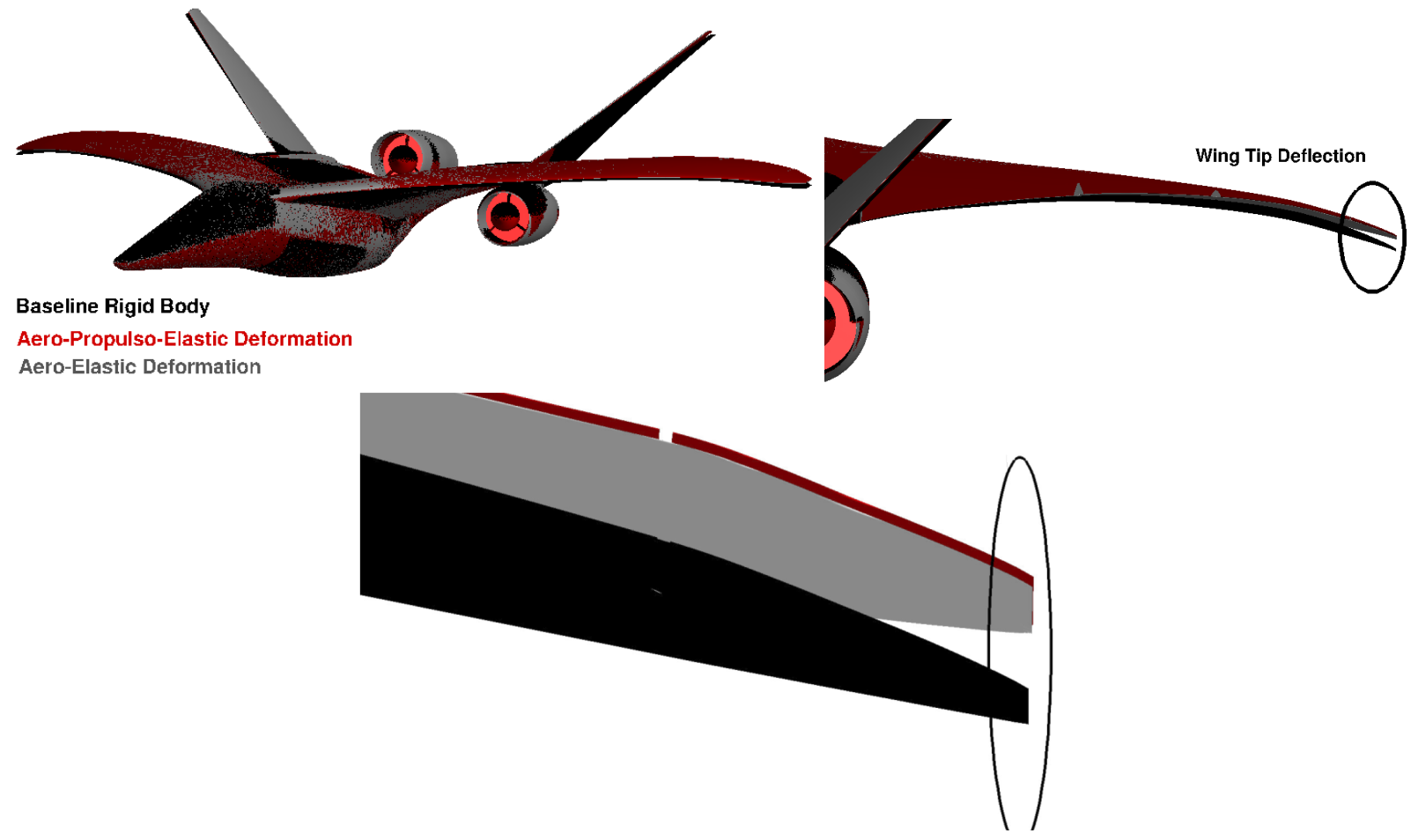

Figure 12. Comparison of baseline, aeroelastic response, and aero-propulso-elastic static aeroelastic response with wing tip deflection.

\section{Future Work}

The problem of the engine simulation restart capability causing some transients at start up needs to be resolved to allow for the static aeroelastic solutions to be obtained while including the engine module. To increase confidence in modeling calculations to capture relevant physics, some mesh refinement techniques will need to be considered. In addition, viscous and turbulence effects should be included to further gain confidence in the computational solution. The envelope of this study for this technology needs to be expanded beyond the cruise condition. Further exploration of the vehicle flight envelope, particularly the traditional problematic aeroelastic flight conditions such as the transonic regime should be investigated. Another course of study will be to investigate the propulsion system boundary conditions sensitivity, which can cause significant movements of the normal shock down the inlet center body cone. This behavior lead to a stronger 
shock profile and caused a deflection at the wing tip of approximately 10". Another course of study should be conducted at off nominal conditions that could perturb the normal shock location to bound potential aero-propulso-elastic impacts.

\section{Conclusion}

This paper presented the development of an aero-propulso-servo-elastic (APSE) model using inviscid computational fluid dynamics (CFD) and linear structural deformations. The APSE model was developed by integrating a previously developed nonlinear dynamic simulation for a variable cycle turbofan engine and an elastic supersonic commercial vehicle transport. The primary focus of this study was to provide a means to include relevant dynamics of a propulsion system into the aeroelastic studies conducted during a vehicle design, which have historically neglected propulsion effects. Static aeroelastic analysis was conducted for comparing the vehicle deformation of the traditional elastic vehicle neglecting the propulsion system against an analysis for a vehicle with the propulsion system. While a steady-state and time-accurate solution could be obtained for the rigid vehicle with the variable cycle engine simulation model, convergence problems prevented such a solution when elastic deformations were considered. Fixed engine boundaries were used to allow for the partial inclusion of the propulsion system impacts while maintaining a stable solution. Static aeroelastic analysis comparisons between the baseline and APSE models for a wing tip deflection indicate that the propulsion system impact on the vehicle elastic response could increase the deflection by $10 \%$. This indicates potential impacts to vehicle design and merits further study.

\section{Acknowledgments}

The authors would like to thank the Fun3D development team for providing assistance and test cases for the interface code between Fun3D and the propulsion system simulation.

\section{References}

\footnotetext{
${ }^{1}$ Silva, W. A. and et al., "Experimental Steady and Unsteady Aerodynamic and Flutter Results for HSCT Semispan Models," AIAA 2000-1697, April 2000.

${ }^{2}$ Connolly, J. W., Kopasakis, G., Carlson, J., and Woolwine, K., "Nonlinear Dynamic Modeling of a Supersonic Commercial Transport Turbo-Machinery Propulsion System for Aero-Propulso-Servo-Elasticity Research," AIAA 2015-4031, July 2015.

${ }^{3}$ Silva, W. A., Sanetrik, M. D., Chwalowski, P., and Connolly, J. W., "Computational Aeroelastic Analysis of a Low-Boom Supersonic Configuration," AIAA 2015-2721, June 2015.

${ }^{4}$ Hodges, D. H., Patil, M. J., and Chae, S., "Effect of Thrust on Bending-Torsion Flutter of Wings," Journal of Aircraft, Vol. 39, No. 2, 2002, pp. 371-376.

${ }^{5}$ Amoozgar, M. R., Irani, S., and Vio, G. A., "Aeroelastic Instability of a Composite Wing with a Powered-Engine," Journal of Fluids and Structures, Vol. 36, January 2013, pp. 70-82.

${ }^{6}$ Firouz-Abadi, R. D., Askarian, A. R., and Zarifian, P., "Effect of Thrust on the Aeroelastic Instability of a Composite Swept Wing with Two Engines in Subsonic Compressible Flow," Journal of Fluids and Structures, Vol. 36, January 2013, pp. 18-31.

${ }^{7}$ Mardanpour, P. and Hodges, D. H., "On the Importance of Nonlinear Aeroelasticity and Energy Efficiency in Design of Flying Wing Aircraft," Advances in Aerospace Engineering, Vol. 2015, No. 613962, 2015, pp. 1-11.

${ }^{8}$ Mazidi, A., Kalantari, H., and Fazelzadeh, S. A., "Aeroelastic Response of an Aircraft Wing with Mounted Engine Subjected to Time-Dependent Thrust," Journal of Fluids and Structures, Vol. 39, No. May, 2013, pp. 292-305.

${ }^{9} \mathrm{McNamara}$, J. J. and Friedmann, P. P., "Aeroelastic and Aerothermoelastic Analysis in Hypersonic Flow: Past, Present, and Future," AIAA Journal, Vol. 49, No. 6, June 2011.

${ }^{10}$ Raney, D. L., McMinn, J. D., Pototzky, A., and Wooley, C. L., "Impact of Aeroelasticity on Propulsion and Longitudinal Flight Dynamics of an Air-Breathing Hypersonic Vehicle," AIAA 1993-1367, 1993.

${ }^{11}$ Rudd, L. V. and Pines, D. J., "Integrated Propulsion Effects on Dynamic Stability and Control of Hypersonic Waveriders," AIAA 2000-3826, July 2000.

${ }^{12}$ Johnson, E. H. and Reymond, M. A., "Multidisciplinary Aeroelastic Analysis and Design Using MSC/NASTRAN," AIAA-1991-1097, April 1991.

${ }^{13}$ Kopasakis, G., Connolly, J. W., and Seidel, J., "Propulsion System Dynamic Modeling of the NASA Supersonic Concept Vehicle for AeroPropulsoServoElasticity," AIAA 2014-3684, July 2014.

${ }^{14}$ Garrard, D., Davis, M., Wehofer, S., and Cole, G., "A One Dimensional, Time Dependent Inlet/Engine Numerical Simulation for Aircraft Propulsion Systems," ASME 97-GT-333, June 1997.

${ }^{15}$ Garrard, D., "ATEC: The Aerodynamic Turbine Engine Code for the Analysis of Transient and Dynamic Gas Turbine Engine System Operations Part 1: Model Development," ASME 96-GT-193, June 1996.
} 
${ }^{16}$ Garrard, D., "ATEC: The Aerodynamic Turbine Engine Code for the Analysis of Transient and Dynamic Gas Turbine Engine System Operations Part 2: Numerical Simulations," ASME 96-GT-194, June 1996.

${ }^{17}$ Gamble, E. J., Haid, D., D'Alessandro, S., and DeFrancesco, R., "Dual-Mode Scramjet Performance Model for TBCC Simulation," AIAA 2009-5298, August 2009.

${ }^{18}$ Numbers, K. and Hamed, A., "Development of a Coupled Inlet-Engine Dynamic Analysis Method," AIAA 1997-2880, July 1997.

${ }^{19}$ Giannola, P., Haas, M., Cole, G., and Melcher, K., "Modeling the Dynamics of Superonic Inlet/Gas-Turbine Engine Systems for Large - Amplitude High-Frequency Disturbances," AIAA 2000-36772, July 2000.

${ }^{20}$ Kopasakis, G., Connolly, J. W., Paxson, D. E., and Ma, P., "Volume Dynamics Propulsion System Modeling for Supersonics Vehicle Research," Journal of Turbomachinery, Vol. 132, No. 4, October 2010, pp. 1-8.

${ }^{21}$ Seldner, K., Mihaloew, J. R., and Blaha, R. J., "Generalized Simulation Technique for Turbojet Engine System Analysis," NASA/TN D-6610, February 1972.

${ }^{22}$ Biedron, R. T. and et al, "FUN3D Manual: 12.7," NASA/TM 2015-218761, May 2015.

${ }^{23}$ Chwalowski, P., Heeg, J., Wieseman, C. D., and Florance, J. P., "FUN3D Analyses in Support of the First Aeroelastic Prediction Workshop," AIAA 2013-0785, 2013.

${ }^{24}$ Anderson, W. K. and Bonhaus, D. L., "An Implicit Upwind Algorithm for Computing Turbulent Flows on Unstructured Grids," Computers and Fluids, Vol. 23, No. 1, 1994, pp. 1-21.

${ }^{25}$ Edwards, J. R., "A Low-Diffusion Flux Splitting Scheme for Navier-Stokes Calculations," Computers and Fluids, Vol. 26, No. 6, July 1997, pp. 653-659.

${ }^{26}$ van Albada, G. D., van Leer, B., and Roberts, W. W. Jr., "A Comparative Study of Computational Methods in Cosmic Gas Dynamics," Astronomy and Astrophysics, Vol. 108, No. 1, April 1982, pp. 76-84.

${ }^{27}$ Vatsa, V. N., Carpenter, M. H., and Lockard, D. P., "Re-evaluation of an Optimized Second Order Backward Difference (BDF2OPT) Scheme for Unsteady Flow Applications," AIAA 2010-0122, Jan. 2010.

${ }^{28}$ Biedron, R. T. and Thomas, J. L., "Recent Enhancements to the FUN3D Flow Solver for Moving-Mesh Applications," AIAA 2009-1360, January 2009.

${ }^{29}$ Bartels, R. E., Rumsey, C. L., and Biedron, R. T., "CFL3D Version 6.4 - General Usage and Aeroelastic Analysis," NASA/TM 2006-214301, April 2006.

${ }^{30}$ Samareh, J. A., "Discrete Data Transfer Technique for Fluid-Structure Interaction," AIAA 2007-4309, June 2007.

${ }^{31}$ Carlson, J., "Inflow/Outflow Boundary Conditions with Application to FUN3D," NASA/TM 2011-217181, October 2011.

${ }^{32}$ Cumpsty, N. A. and Horlock, J. H., "Averaging Nonuniform Flow for a Purpose," Journal of Turbomachinery, Vol. 128, No. 1, February 2005, pp. 120-129. 